\title{
The HPA/SDC1 axis promotes invasion and metastasis of pancreatic cancer cells by activating EMT via FGF2 upregulation
}

\author{
XIDONG CHEN $^{1,2}$, HAICHAO ZHAO ${ }^{1,2}$, CHANGZHOU CHEN $^{1,2}$, JIAN LI ${ }^{1,2}$, \\ JIEFENG $\mathrm{HE}^{2}$, XIFENG FU ${ }^{2}$ and HAOLIANG ZHAO ${ }^{1,2}$ \\ ${ }^{1}$ Graduate School, Shanxi Medical University, Taiyuan, Shanxi 030000; \\ ${ }^{2}$ Department of General Surgery, Shanxi Dayi Hospital Affiliated to Shanxi Medical University, \\ Taiyuan, Shanxi 030032, P.R. China
}

Received May 4, 2019; Accepted October 3, 2019

DOI: $10.3892 / \mathrm{ol} .2019 .11121$

\begin{abstract}
Pancreatic cancer is characterized by the absence of early specific clinical symptoms, accompanied with rapid metastasis and invasion. It is one of the most prevalent types of cancer and more importantly, one of the most common types of malignant cancer with the highest mortality rate of all cancer types. The heparanase (HPA)/syndecan-1 (SDC1) axis has been reported to promote tumor growth, invasion, metastasis and angiogenesis in a variety of cancer types; however, studies into the role and mechanism of the HPA/SDC1 axis in pancreatic cancer are limited. The present study aimed to investigate the biological function and clinical significance of the HPA/SDC1 axis in pancreatic cancer. The results demonstrated that HPA is elevated in pancreatic cancer tissues and cell lines, and that its high expression was associated with poor prognosis. HPA was revealed to mediate an increase in fibroblast growth factor 2 (FGF2) expression by upregulating the expression of SDC1. Conversely, silencing HPA mediated the suppression of FGF2 expression. Furthermore, upregulated FGF2 was observed to increase the expression of downstream Palladin proteins by activating the PI3K/Akt signaling pathway and also lead to the activation of epithelial-mesenchymal transition (EMT). Subsequently, EMT was found to promote the migration and invasion of pancreatic cancer cells. In summary, the HPA/SDC1 axis was revealed to serve an important role in the regulation of FGF2, and was found to promote the invasion and metastasis of pancreatic cancer cells. These findings indicated that the HPA/SDC1 axis may be used as an effective therapeutic target for pancreatic cancer.
\end{abstract}

Correspondence to: Professor Haoliang Zhao, Department of General Surgery, Shanxi Dayi Hospital Affiliated to Shanxi Medical University, 99 Longcheng Street, Taiyuan, Shanxi 030032, P.R. China

E-mail: zhaohlxueshu@163.com

Key words: pancreatic cancer, heparanase, fibroblast growth factor 2 , syndecan-1, epithelial-mesenchymal transition

\section{Introduction}

According to the most recent global cancer data, there were $\sim 458,900$ new cases of pancreatic cancer worldwide in 2018 and 432,200 deaths. The mortality rate of pancreatic cancer is the fourth highest in the USA and other Western countries compared with all other types of cancer (1). In recent years, various therapeutic strategies against pancreatic cancer, including surgical resection and targeted therapy, have improved (2). However, due to early metastasis and invasion of pancreatic cancer, only $15-20 \%$ of patients are able to undergo surgical resection after diagnosis. Furthermore, this treatment method has not been shown to improve the prognosis of the remaining patients, as surgical resection does not significantly improve the condition of patients (3). Consequently, improved understanding the mechanisms underlying pancreatic cancer metastasis and invasion is essential.

As previously reported, syndecan-1 (SDC1) is a cell adhesion molecule expressed on the cell surface, which is usually characterized by covalent bonds of heparan sulfate (HS) (4). Ibrahim et al (5) reported the involvement of SDC1 in breast cancer, suggesting that this protein maintains cell phenotype and inhibits cell migration, whereas when SDC1 is isolated from the cell surface, cells may exhibit enhanced proliferative and migratory abilities. Heparanase (HPA) is an endoglycosidase present in mammals that is able to specifically degrade the HS side chain of SDC1. It has been observed that HPA may degrade this side chain in a variety of tumor cells to form the HPA/SDC1 axis (6). Increased expression of HPA in pancreatic cancer cells may disrupt the extracellular matrix (ECM) and basement membrane (BM), thus creating favorable conditions for the invasion and migration of pancreatic cancer cells $(7,8)$. Recently, the role of fibroblast growth factor 2 (FGF2) in the development and progression of malignant cancer types has attracted great interest (9). The binding of FGF2 to its receptor, fibroblast growth factor receptor 2, may lead to the activation of tumor-related signaling pathways, thus increasing cell proliferation, migration and invasion (10).

Masola et al (11) previously reported that HPA may promote the process of renal fibrosis by upregulating the expression of FGF2. In the present study, the expression of the HPA/SDC1 axis and FGF2 in pancreatic cancer cells was analyzed, and 
the mechanisms of their interactions, and more importantly, their effects on epithelial-mesenchymal transition (EMT) and pancreatic cancer progression were the main areas of focus.

\section{Materials and methods}

Clinical specimens. A total of 62 primary pancreatic cancer tissues and 20 adjacent normal tissues located $>2 \mathrm{~cm}$ away from cancer tissues were obtained from patients (38 males and 24 females; age range, 38-72 years; median age, 58.65 years) who underwent surgical resection at Shanxi Dayi Hospital Affiliated to Shanxi Medical University between January 2016 and June 2018. All tissue specimens were examined by pathologists and diagnosed as pancreatic ductal adenocarcinomas. No antineoplastic treatment was administered to the patients prior to the operation. The present study was reviewed and ethically approved by the Shanxi Dayi Hospital Ethics Committee. Informed written consent was obtained from each patient and his/her family.

Cell culture. Human pancreatic cancer cell lines (SW1990, PANC-1, BxPC-3 and Aspc-1) and human pancreatic ductal epithelial cell line (HPDE6c7) were from the American Type Culture Collection. All cell lines were cultured in RPMI 1640 medium (Gibco; Thermo Fisher Scientific, Inc.) containing $100 \mathrm{U} / \mathrm{ml}$ penicillin and $100 \mathrm{U} / \mathrm{ml}$ streptomycin, supplemented with $10 \%$ FBS (Gibco; Thermo Fisher Scientific, Inc.) in a humidified atmosphere of $5 \% \mathrm{CO}_{2}$ at $37^{\circ} \mathrm{C}$.

Reverse transcription-quantitative polymerase chain reaction (RT-qPCR) analysis. Total RNA of pancreatic cancer cells was extracted using TRIzol ${ }^{\circledR}$ reagent (Takara Biotechnology Co., Ltd.) according to the instructions of the manufacturer. A PrimeScript RT Reagent kit (Takara Biotechnology Co., Ltd.) was used for the reverse transcription of mRNA into cDNA at $37^{\circ} \mathrm{C}$ for $60 \mathrm{~min}$ and $85^{\circ} \mathrm{C}$ for $1 \mathrm{~min}$. Gene amplification was then performed. qPCR was performed using a SYBR PrimeScript RT-PCR Kit (Takara Biotechnology Co., Ltd.) according to the instructions of the manufacturer. The reaction started at $95^{\circ} \mathrm{C}$ for $5 \mathrm{~min}$, followed by 35 cycles of $95^{\circ} \mathrm{C}$ for $45 \mathrm{sec}, 59^{\circ} \mathrm{C}$ for $30 \mathrm{sec}$, and $72^{\circ} \mathrm{C}$ for $45 \mathrm{sec}$. The quantification was performed using the $2^{-\Delta \Delta C q}$ method (12), and $\beta$-actin was used as the internal reference gene to normalize the results. The primer sequences are presented in Table I.

Vector construction and cell transfection. The PANC-1 cell line $\left(2 \times 10^{5}\right.$ cells/well) in logarithmic growth phase was inoculated in a 6-well plate for $24 \mathrm{~h}$. HPA silencing vectors were constructed using three short hairpin (sh)RNAs (50 nM; Shanghai GeneChem Co., Ltd; Table II) specifically targeting HPA. The HPA overexpression vector was constructed using a recombinant plasmid encoding HPA $(50 \mathrm{nM}$; cat. no. GV147; Shanghai GeneChem Co., Ltd.), and the empty vector was used as a negative control. The PANC-1 cell line was then transfected to construct a silent (shHPA group) or overexpressed (HPA group) cell line. The PANC-1 cell line was transfected with an empty vector containing the GFP sequence as a control (vector group). FGF2 silencing vectors were constructed using (sh)RNA (5'-ACTACAATACTT ACCGGTCAA-3') (50 nM; Shanghai GeneChem Co., Ltd)
Table I. Sequences of primers.

\begin{tabular}{llc}
\hline Gene & \multicolumn{1}{c}{\begin{tabular}{c}
\multicolumn{1}{c}{ Primer } \\
sequence $\left(5^{\prime} \rightarrow 3^{\prime}\right)$
\end{tabular}} & $\begin{array}{c}\text { Amplicon } \\
\text { size, bp }\end{array}$ \\
\hline HPA & $\begin{array}{l}\text { F: GAATGGACGGACTGCTAC } \\
\text { R: CCAAAGAATACTTGCCTCA }\end{array}$ & 261 \\
FGF2 & $\begin{array}{l}\text { F: AGCGGCTGTACTGCAAAAAC } \\
\text { R: CCCAGGTCCTGTTTGGAT }\end{array}$ & 338 \\
Palladin & F: GCATCAGAGCTGACCTCAAC \\
& $\begin{array}{l}\text { R: GGTTGATCACCGACGCTAAT } \\
\text { Akt }\end{array}$ & $\begin{array}{l}\text { F: GCGACAGTGGCTATTGTGA } \\
\text { R: AGCTGCTCAAAGATGC CATT }\end{array}$ \\
\multirow{2}{*}{ SDC1 } & $\begin{array}{l}\text { F: AGCTGACCTTCACACTCC } \\
\text { R-actin }\end{array}$ & $\begin{array}{l}\text { R: TCGGCTCCTCCAAGGAGT } \\
\text { F: GTCAGTGCATCACTACTGGCAAT }\end{array}$ \\
& R: CGATCTGGTAG GGCCTTG & 369 \\
\end{tabular}

HPA, heparanase; FGF2, fibroblast growth factor 2; SDC1, syndecan-1; F, forward; R, reverse.

specifically targeting FGF2. The PANC-1 cell line was then transfected to construct a silent (shFGF2 group) cell line. The SDCI inhibitor synstatin (100 nM; Beyotime Institute of Biotechnology) was added to the HPA group to form the HPA + synstatin group. The FGF2 receptor (FGF2R) inhibitor AZD4547 and the PI3K/Akt-specific inhibitor LY294002 were purchased from Sigma-Aldrich; Merck KGaA. PANC-1 cells were divided into the following groups: i) FGF2 group [PANC-1 cell line transfected with FGF2 recombinant plasmid (100nM; cat. no. QCP5411; QCHENG BIO Inc.)]; ii) AZD4547 group (3.0 nmol-1 AZD4547 added to the FGF2 group); iii) LY294002 group (10 $\mu$ mol-1 LY294002 added to the FGF2 group); and iv) control group [PANC-1 cell line transfected with empty plasmid (cat. no. QCP5400; QCHENG BIO Inc.]. Transfection was performed using Lipofectamine ${ }^{\circledR}$ 3000 (Invitrogen; Thermo Fisher Scientific, Inc.), according to the instructions of the manufacturer. Subsequent experiments were performed $48 \mathrm{~h}$ after transfection.

Immunohistochemical staining. Tumor tissues were fixed with $10 \%$ formalin solution for $15 \mathrm{~min}$ at room temperature, and then blocked in wash buffer containing 5\% normal goat serum (Shanghai GeneChem Co., Ltd.) for $2 \mathrm{~h}$ at room temperature. Tumor tissues were incubated with rabbit anti-human HPA primary antibody (1:100; cat. no. PB0405) and rabbit anti-human FGF2 primary antibody (1:100; cat. no. PB0916; both Boster Biological Technology) at $4^{\circ} \mathrm{C}$ overnight, and then incubated with goat anti-rabbit IgG secondary antibody (1:200; cat. no. BA1003; Boster Biological Technology) for $2 \mathrm{~h}$ at room temperature. The tissue was then stained with diaminobenzidine, and the nuclei were stained with hematoxylin for $5 \mathrm{~min}$ at room temperature and then observed using an optical microscope (Olympus Corporation) at x200 magnification. Immunohistochemical staining was performed according to the protocols of the manufacturer (Beijing Zhongshan Golden Bridge Biotechnology Co., Ltd.; OriGene Technologies, 
Table II. Target sequences of shHPA.

\begin{tabular}{llrr}
\hline ID & Target sequence $\left(5^{\prime} \rightarrow 33^{\prime}\right)$ & Initial position & GC content $(\%)$ \\
\hline sh1 & GCTCTGTAGATGTGCTATACA & 640 & 42.86 \\
sh2 & GCATCACTACTATTTGAATGG & 1022 & 38.1 \\
sh3 & GCTTGCCAGCTTTCTCATATA & 1705 & 42.86 \\
\hline
\end{tabular}

GN, guanine-cytosine.

A

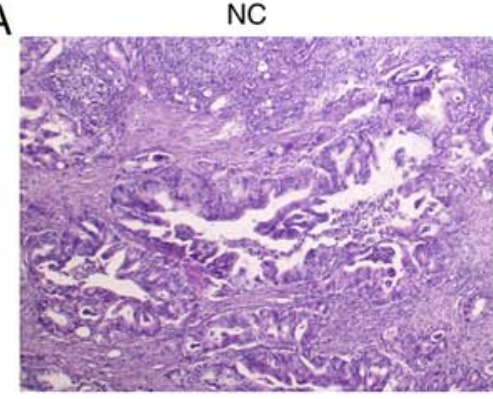

HPA

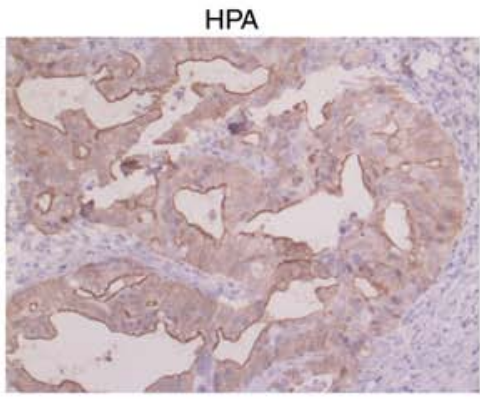

NC

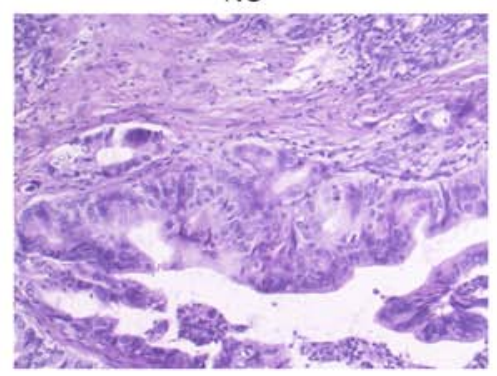

FGF2

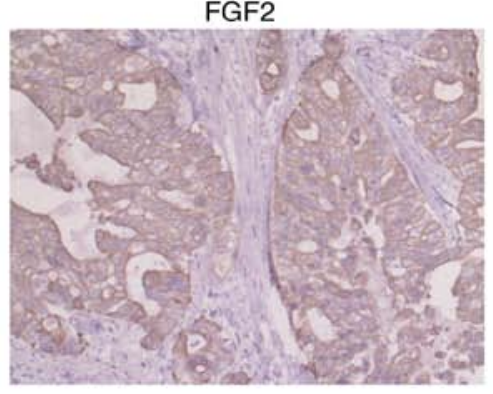

B
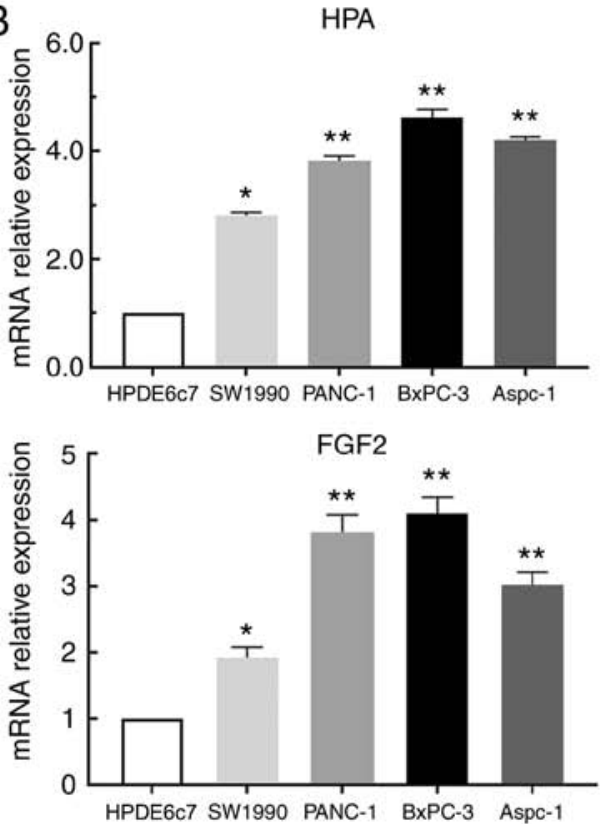

Figure 1. HPA and FGF2 are upregulated in pancreatic cancer tissues and cell lines. (A) Comparison of HPA and FGF2 expression in pancreatic cancer tissues and adjacent normal pancreatic tissues by immunohistochemical stainin. (B) Analysis of relative mRNA expression levels of HPA and FGF2 in pancreatic cancer and normal pancreatic cell lines by reverse transcription-quantitative PCR. ${ }^{*} \mathrm{P}<0.05,{ }^{* *} \mathrm{P}<0.01$ vs. HPDE6c7. HPA, heparanase; FGF2, fibroblast growth factor 2; NC, negative control.

Inc.). The following scoring system was used to evaluate the staining results: The staining intensity of HPA and FGF2 was rated $0-3$ ( 0 , negative; 1 , weak; 2 , moderate; or 3 , strong), while marker expression was evaluated according to the percentage of the stained area: $0,0-10 \% ; 1,11-25 \% ; 2,26-50 \%$; or 3 , $50-100 \%$. The final result was calculated as the sum of the two scores. The rating criteria were as follows: 0-3, low expression; and 4-6, high expression. The results were evaluated by two pathologists and the differences were re-evaluated until a consensus was reached.

Western blot analysis. Total protein was extracted by lysing the cells with RIPA buffer (Beyotime Institute of Biotechnology) and the protein concentration was measured using a BCA protein assay kit (Beyotime Institute of Biotechnology). A total of $50 \mu \mathrm{g}$ protein from each sample was separated by SDS-PAGE (10\% gel) and the proteins were transferred to a nitrocellulose membrane by electrophoretic transfer. After blocking with 5\% skimmed milk for $2 \mathrm{~h}$ at room temperature, the membranes were incubated with the following primary antibodies: Rabbit anti-human HPA (1:1,000; cat. no. PB0405), rabbit anti-human FGF2 (1:1,000; cat. no. PB0916), rabbit anti-human AKT
(1:5,000; cat. no. A00024-1), rabbit anti-human E-cadherin (1:100; cat. no. BA0475), rabbit anti-human N-cadherin (1:100; cat. no. BM3921), rabbit anti-human vimentin 1:3,000 (cat. no. PB0378; all Boster Biological Technology) and rabbit anti-human palladin $(1: 100$; cat. no. PA5-65160; Thermo Fisher Scientific Inc.) were added according to the instructions of the manufacturer and incubated at $4^{\circ} \mathrm{C}$ overnight. $\beta$-actin (1:500; cat. no. BA2305; Boster Biological Technology) was used as an internal loading control. The goat anti-rabbit IgG secondary antibody (1:5,000; cat. no. BA1056; Boster Biological Technology) labeled with horseradish peroxidase was added to the membrane after washing and incubated at room temperature. The gray value of the protein bands was analyzed by ImageJ software (v1.8.0; National Institutes of Health) and the ratio of the gray value of the target band to that of the internal reference $\beta$-actin was representative of the expression level of the protein. All assays were performed in triplicate.

Transwell chamber assay. The invasive ability of PANC-1 cells was measured using Transwell chambers (Corning Inc.). PANC- 1 cells $\left(2 \times 10^{5}\right.$ cells $\left./ \mathrm{ml}\right)$ starved for $24 \mathrm{~h}$ were inoculated 
Table III. Expression of heparanase and fibroblast growth factor 2 in pancreatic cancer tissues and adjacent normal tissues.

\begin{tabular}{|c|c|c|c|c|c|c|}
\hline Parameter & Patients, $\mathrm{n}$ & Positive & Negative & Positive rate $(\%)$ & $\chi^{2}$-value & P-value \\
\hline HPA & & & & & 31.236 & $<0.001$ \\
\hline Tumor & 62 & 47 & 15 & 75.806 & & \\
\hline Non-tumor & 20 & 1 & 19 & 5.000 & & \\
\hline FGF2 & & & & & 32.529 & $<0.001$ \\
\hline Tumor & 62 & 50 & 12 & 80.645 & & \\
\hline Non-tumor & 20 & 2 & 18 & 10.000 & & \\
\hline
\end{tabular}

HPA, heparanase; FGF2, fibroblast growth factor 2; Tumor, tumor tissues; Non-tumor, adjacent non-tumor tissues.

into the upper chamber of a 24-well chamber, and each group was analyzed in 6 replicate wells. A total of $50 \mu$ l Matrigel solution was placed on the polycarbonate microporous membrane between the upper and the lower chambers, $100 \mu 1$ RPMI 1640 medium was added to the upper chamber, and a mixture of $600 \mu 1$ RPMI 1640 and $20 \%$ fetal bovine serum was added to the lower chamber. After incubation for $24 \mathrm{~h}$ at room temperature, the cells were fixed with a $4 \%$ paraformaldehyde solution for $30 \mathrm{~min}$ at room temperature, followed by rinsing with PBS and staining with a $0.5 \%$ crystal violet dye solution for $10 \mathrm{~min}$ at room temperature. Subsequently, the lower chamber was observed and photographed with a light microscope at x200 magnification, and cell counting was performed.

Wound healing assay. PANC-1 cells were seeded in a 6-well plate at an adjusted concentration of $1 \times 10^{6}$ cells $/ \mathrm{ml}$. The experiment was performed when the cells reached $100 \%$ confluence. A wound was created in the center of the plate with the tip of a $100-\mathrm{ml}$ pipette. The scratched cells were washed away in serum-free medium and this process was repeated 3 times, and then cultured in regular medium. The wound was observed at x200 magnification with an inverted microscope at 0 and $48 \mathrm{~h}$, and images were captured.

Statistical analysis. SPSS software (version 23.0; IBM Corp.) was used for statistical analysis throughout the study. Statistical analysis of HPA and FGF2 expression between pancreatic cancer tissues and paired adjacent normal tissues was evaluated using a paired Student's t-test. One-way analysis of variance was performed on $\geq 3$ sets of data using Tukey's post hoc test. GraphPad Prism software (version 8.0; GraphPad Software, Inc.) was used to generate graphical representations. Each experiment was repeated $\geq 3$ times. The experimental results are presented as mean \pm SD values. $\mathrm{P}<0.05$ was considered to indicate a statistically significant difference.

\section{Results}

HPA and FGF2 are upregulated in pancreatic cancer tissues and cells. In order to investigate the expression of HPA and FGF2 in pancreatic cancer tissues and cells, immunohistochemical staining and RT-qPCR analysis were performed. According to the results of immunohistochemistry, significant upregulation of HPA and FGF2 was observed in pancreatic cancer tissues compared with adjacent non-tumor tissues
(Fig. 1A). Based on the aforementioned scoring criteria, the details of HPA and FGF2 expression are presented in Table III. The results of RT-qPCR analysis revealed that HPA and FGF2 expression levels were significantly higher in pancreatic cancer cell lines than in the HPDE6c7 cell line (Fig. 1B). These results implied that the expression of HPA and FGF2 in pancreatic cancer was markedly upregulated.

Association of HPA and FGF2 expression with clinicopathological characteristics of patients with pancreatic cancer. To further explore whether elevated HPA and FGF2 expression levels are directly associated with the clinicopathological characteristics of pancreatic cancer, the expression of HPA and FGF2 in different pancreatic cancer tissues was examined via immunohistochemistry. The results revealed that the positive rate of HPA in tissues with lymph node metastasis was significantly higher than that in tissues without lymph node metastasis. Tumor-node-metastasis (TNM) stage III-IV tissues were found to have higher HPA-positive expression than TNM stage I-II tissues. Positive expression of HPA was not associated with the age, sex, tumor location or tumor differentiation status of patients with pancreatic cancer. By contrast, the positive expression rate of FGF2 was observed to increase as the degree of tumor differentiation decreased. Moreover, similarly to HPA, the age, sex or tumor location of the patients with pancreatic cancer were not associated with FGF2 expression (Table IV).

HPA is capable of upregulating the expression of FGF2 in pancreatic cancer cells. According to the aforementioned experimental results, the expression of HPA and FGF2 in pancreatic cancer cells was significantly increased. For the purpose of determining whether the expression of FGF2 was affected by HPA, the expression of FGF2 in the shHPA, HPA and vector groups was compared. Among the three PANC-1 shHPA cell lines selected, the sh1 group had the highest silencing efficiency, compared with the sh2 and sh3 groups (Fig. 2A and B). The RT-qPCR and western blot analyses demonstrated that the expression of FGF2 was significantly increased in the HPA group compared with the vector group. On the contrary, HPA silencing significantly inhibited the expression of FGF2 in the shHPA group compared with the vector group (Fig. 2C and D). Taken together, these results suggested that HPA is able to increase the expression of FGF2, and that HPA silencing results in the downregulation of FGF2 in pancreatic cancer cells. 


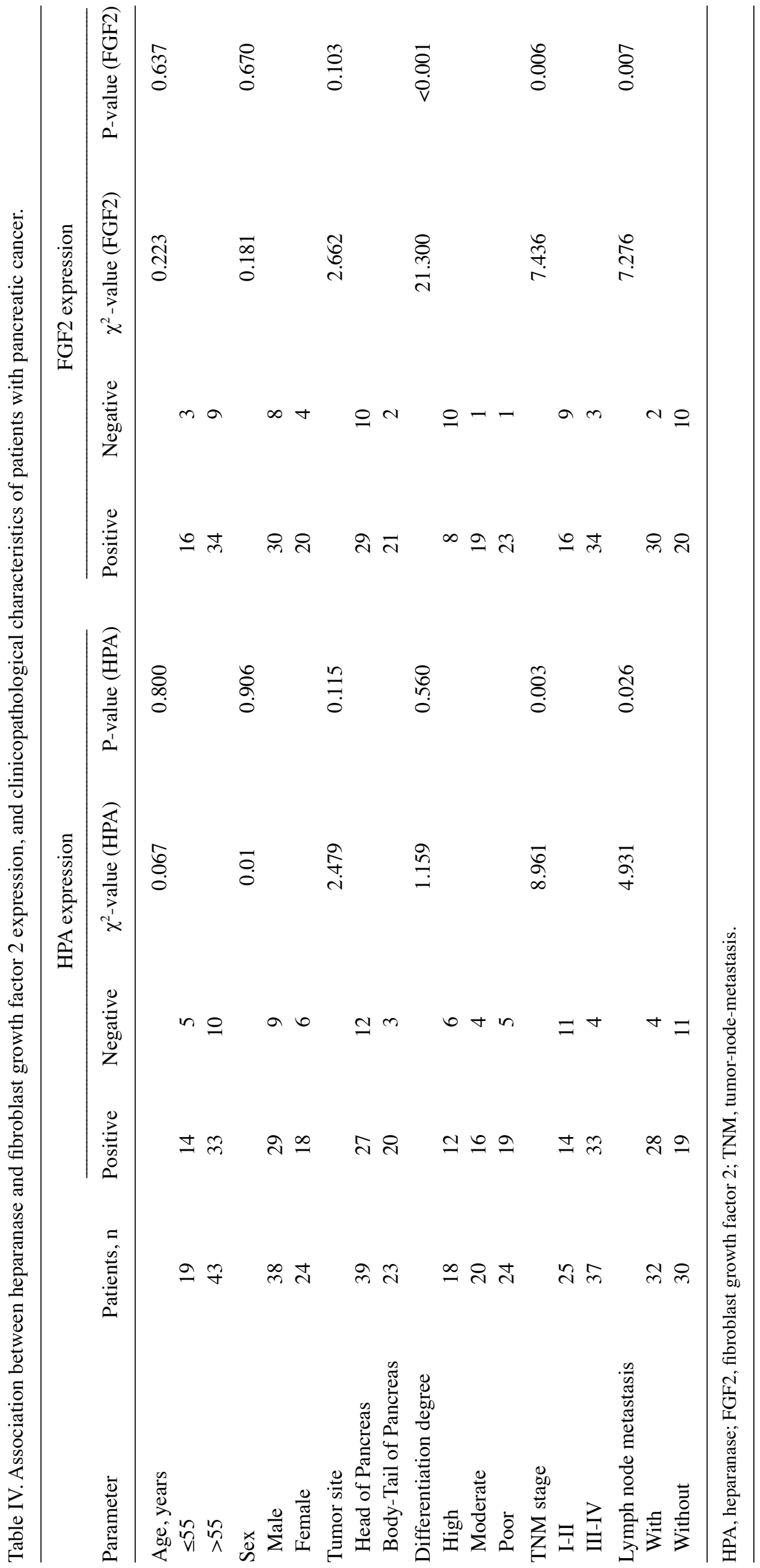



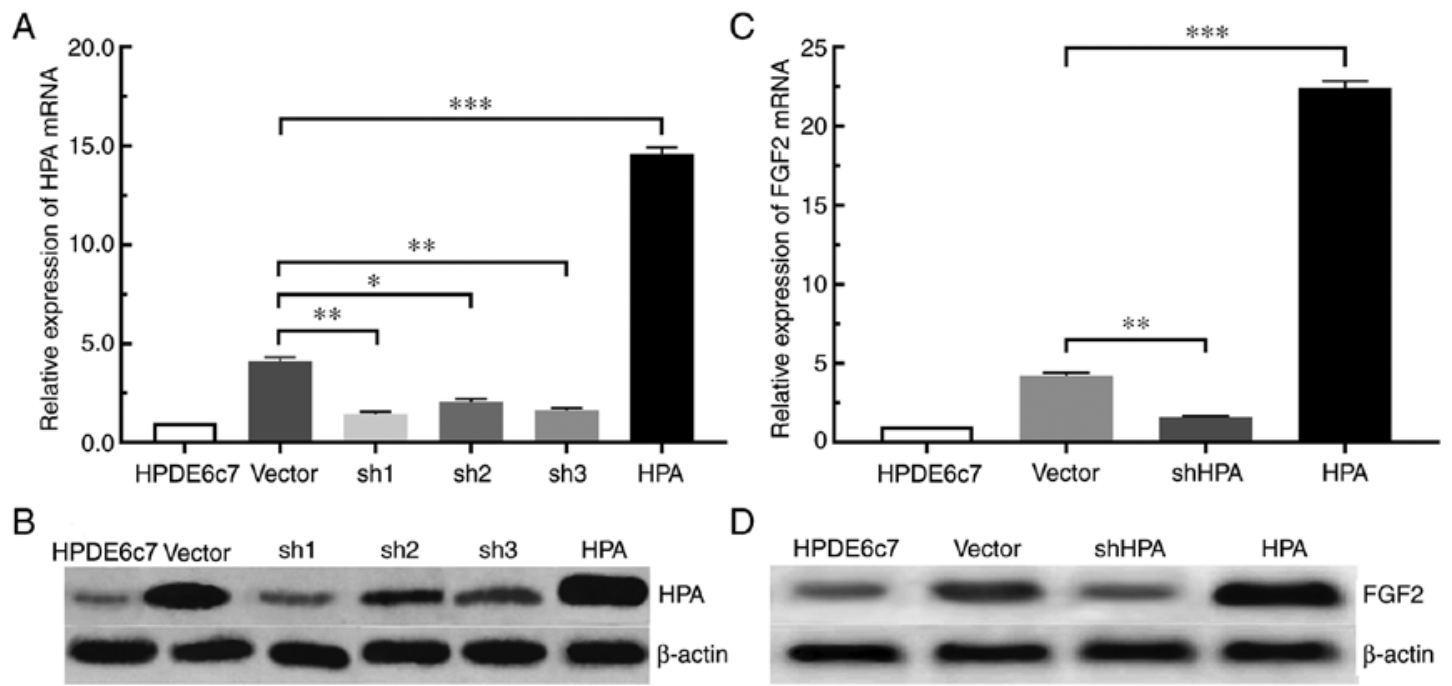

Figure 2. HPA upregulates the expression of FGF2 in pancreatic cancer cell lines. (A) Analysis of HPA silencing and overexpression efficiency by RT-qPCR (B) Analysis of HPA silencing and overexpression efficiency by western blot assay. (C) Analysis of FGF2 expression after silencing or overexpressing HPA by RT-qPCR. (D) Analysis of FGF2 expression after silencing or overexpressing HPA by western blotting. $\beta$-actin was used as an internal control. "P<0.05, ${ }^{* *} \mathrm{P}<0.01,{ }^{* * *} \mathrm{P}<0.001$. RT-qPCR, reverse transcription-quantitative PCR; HPA, heparanase; FGF2, fibroblast growth factor 2; sh, short hairpin (RNA; specific for HPA); Vector, PANC-1 cell line transfected with empty plasmid containing the GFP sequence.

HPA regulates FGF2 expression via the HPA/SDC1 axis in pancreatic cancer cells. By binding to SDC1, HPA removes this proteoglycan from the cell surface and promotes its expression (13). To confirm the study hypothesis that HPA affects the expression of FGF2 in pancreatic cancer cells by binding to SDC1, RT-qPCR was performed. The results revealed that SDC1 expression was increased in pancreatic cancer cell lines compared with the HPDE6c7 cell line. (Fig. 3A). Overexpression of HPA resulted in a significant increase in SDC1 expression in the HPA group compared with the vector group, whereas HPA silencing considerably suppressed the expression of SDC1 (Fig. 3B). Moreover, the expression of FGF2 was markedly decreased in the HPA + synstatin group compared with that in the vector group (Fig. 3C). Taken together, these findings indicated that HPA may promote the expression of FGF2 by upregulating SDC1 in pancreatic cancer cells. Notably, neither overexpression nor silencing of FGF2 inflenced HPA expression (Fig. S3)

HPA promotes pancreatic cancer cell migration and invasion by upregulating FGF2 to activate the PI3K/Akt pathway and EMT process.

Increased expression of Palladin in pancreatic cancer tissues and cells. A previous study demonstrated that Palladin is highly expressed in pancreatic cancer cells (14). Moreover, the PI3K/Akt pathway has been observed to maintain the stability of Palladin and promote its expression (15). It was hypothesized that FGF2 promotes the expression of Palladin via the PI3K/Akt pathway, thereby promoting the invasion and migration of pancreatic cancer cells. According to the western blot results, the expression of Palladin in pancreatic cancer cells was significantly higher than in the HPDE6c7 cell line (Fig. 4A). This result was further confirmed via RT-qPCR analysis (Fig. 4B).

FGF2 promotes pancreatic cancer cell invasion and migration via the PI3K/Akt pathway. Successful overexpression of FGF2 in FGF2 group cells was determined via RT-qPCR and western blot analyses (Fig. S1). According to the western blot analysis, the expression of Palladin and Akt proteins in the FGF2 group was significantly increased compared with the other groups (Fig. 4C and D). This result was also confirmed by RT-qPCR (Fig. S2). The invasive and migratory abilities of pancreatic cancer cells were assessed by Transwell chamber and wound healing assays. When FGF2 was overexpressed in the FGF2 group, the invasive and migratory abilities of pancreatic cancer cells were significantly enhanced compared with the control group. However, these abilities of pancreatic cancer cells were markedly reduced in the AZD4547 and LY294002 groups compared with in the FGF2 and control groups. (Fig. 4E-G). These results indicated that FGF2 may regulate the invasion and migration of pancreatic cancer cells via the PI3K/Akt signaling pathway, thus promoting the progression of pancreatic cancer.

HPA is able to activate the EMT process by upregulating $F G F 2$. EMT is closely associated with cell migration and invasion. To examine the effects of HPA and FGF2 on EMT, western blot analysis was conducted in order to detect the markers of EMT. The expression of E-cadherin was found to be inhibited, whereas the expression of $\mathrm{N}$-cadherin and vimentin was considerably increased after HPA overexpression; these effects were reversed by adding AZD4547 (Fig. 4H). These results demonstrated that HPA may activate the EMT process by upregulating the expression of FGF2.

\section{Discussion}

ECM and BM degradation is a prerequisite for tumor metastasis (16). The secretion of HPA is important for tumors to break down ECM and BM (7). HPA is the only endoglycosidase in the human body that is able to degrade the HS chain in glycosaminoglycans (8). It specifically recognizes sites on the HS side chain and cleaves HS, resulting in the formation of short 

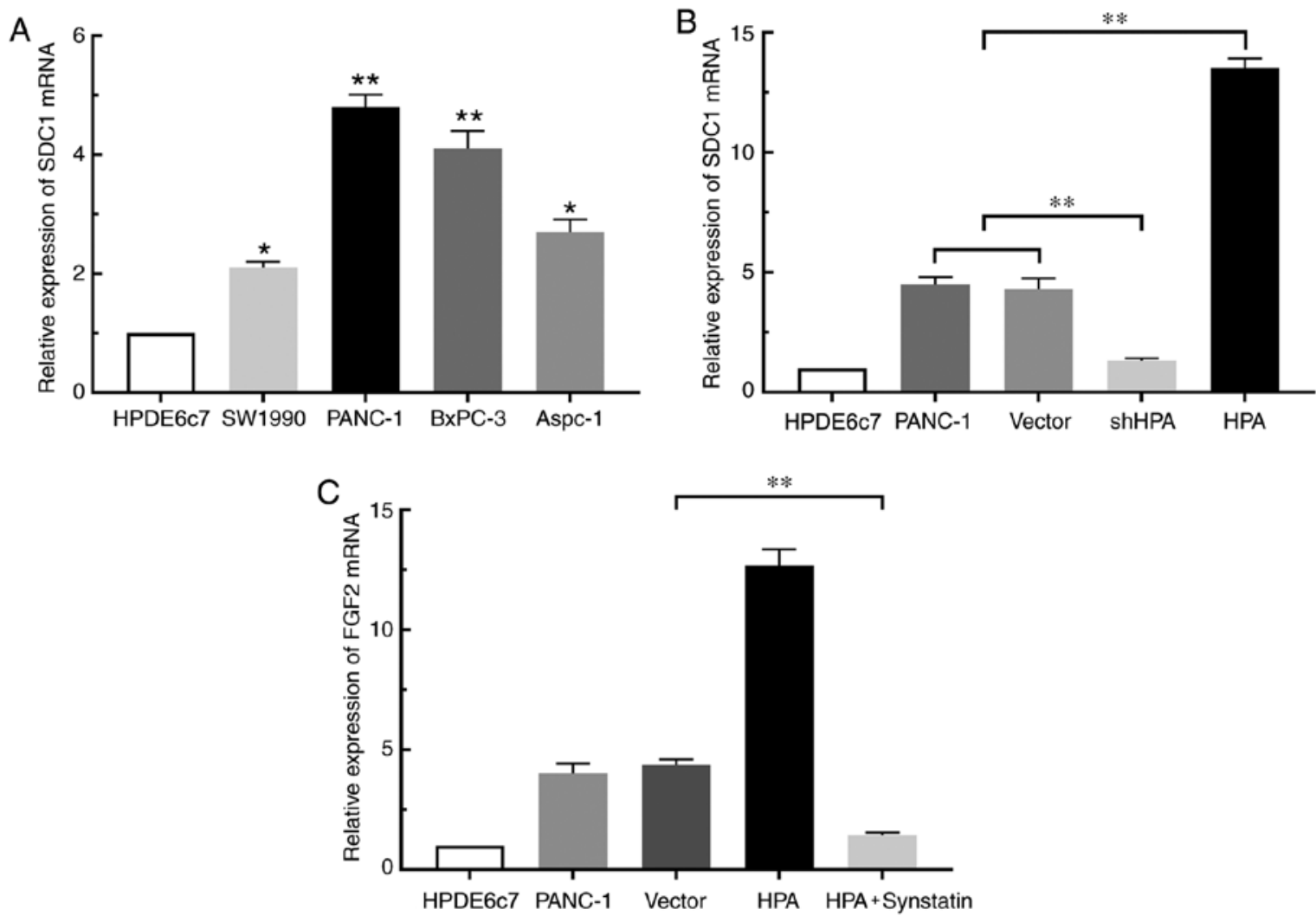

Figure 3. HPA regulates FGF2 expression via the HPA/SDC1 axis in pancreatic cancer cell lines. (A) RT-qPCR comparing the relative expression levels of SDC1 mRNA in pancreatic cancer lines and the normal pancreatic cell line HPDE6c7. ${ }^{*} \mathrm{P}<0.05,{ }^{* * *} \mathrm{P}<0.01$ vs. HPDE6c7. (B) Detection of the effect of HPA on SDC1 mRNA expression by RT-qPCR. (C) Expression of FGF2 was determined by RT-qPCR analysis after adding the SDC1 inhibitor synstatin to the HPA overexpression group. ${ }^{* *} \mathrm{P}<0.01$. RT-qPCR, reverse transcription-quantitative PCR; HPA, heparanase; FGF2, fibroblast growth factor 2; sh, short hairpin (RNA); SDC1, syndecan-1; Vector, PANC-1 cell line transfected with empty plasmid containing the GFP sequence.

fragments with a length of 10-20 sugar units, which release certain growth factor (including fibroblast growth factor and hepatocyte growth factor) bound to HS, thereby promoting tumor invasion and metastasis $(16,17)$. HPA-mediated tumor metastasis may be influenced by a number of factors, including the following: i) Dysregulation of HPA may mediate the release of angiogenic factors including vascular endothelial growth factor and cyclooxygenase-2, thus facilitating the regeneration of tumor microenvironment vessels $(18,19)$; ii) HPA promotes fibrosis, and regulates the expression of transforming growth factor- $\beta$ and other factors, creating a hypoxic microenvironment that is conducive to tumor growth and resulting in degradation of the normal tissue structure (while preventing drug uptake), further promoting the chemoresistance of tumors due to the fibrosis surrounding them (20); and iii) HPA cleaves the HS proteoglycan, consequently destroying the normal structure of ECM and BM, which are protective structures that hinder tumor cell migration (21). In addition, HPA may enhance tumor invasion and metastasis via various mechanisms, which include promoting the progression of inflammation and the formation of exosomes and increasing the chemical resistance of tumors $(22,23)$.

In recent years, the role of growth factors in the progression of tumors is an area that has gained great interest $(24,25)$. After binding to their specific receptors, growth factors may affect a series of biological activities by activating downstream signaling pathways (26). As a member of the growth factor family, FGF2 has been detected in several types of cancer, including breast and gastric cancer, and FGF2 is highly expressed in these types of cancers $(27,28)$. A limited number of studies have also reported the detection of FGF2 in pancreatic cancer $(29,30)$. The present study demonstrated that HPA and FGF2 were highly expressed in pancreatic cancer tissues and cell lines. HPA overexpression resulted in increased FGF2 expression. A study by Li et al (31) also confirmed the present study's hypothesis; it revealed that in rheumatoid arthritis, upregulation of HPA can significantly promote the expression of FGF2. Conversely, HPA silencing was observed to mediate a reduction of FGF2. The present study also analyzed the impact of FGF2 dysregulation on HPA. Notably, neither overexpression nor silencing of FGF2 had an effect on HPA expression, a result that differed from the findings reported by Masola et al (11). Masola et al believe that HPA can promote the expression of FGF2 in renal fibrosis, and the high expression of FGF2 can further promote the expression of HPA. This difference may be due to the pancreatic cancer cell lines selected for the present study. Kato et al (32) revealed that HPA is capable of converting SDC1 into an activator of FGF2 in certain cases. It was hypothesized that HPA regulated FGF2 via SDC1 in pancreatic cancer. In the PANC-1 cell line, the expression level of SDC1 increased when HPA was overexpressed, whereas HPA silencing had the opposite effect. SDC1 inhibitors were added to the HPA overexpression group to detect the effect of SDC1 on FGF2, and it was observed that 
A

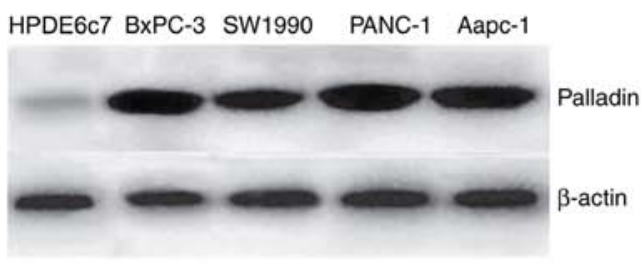

C

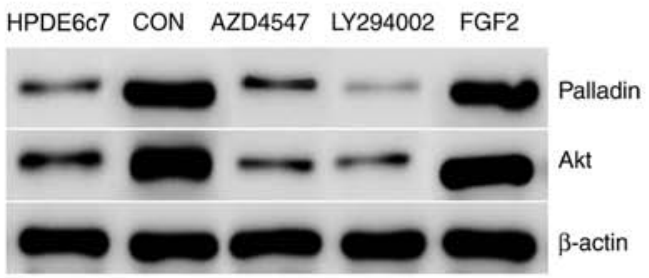

$\mathrm{B}$
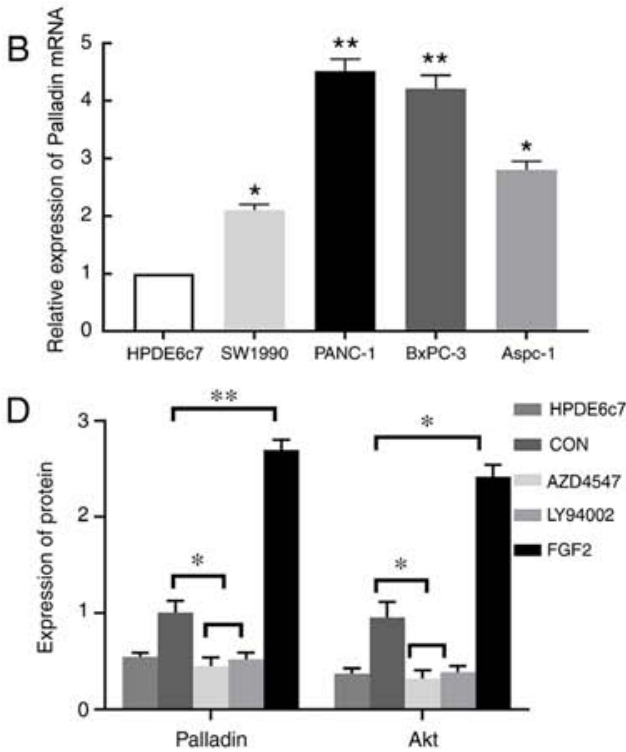

$\mathrm{E}$

CON FGF2 AZD 4547 LY294002

$\mathrm{F}$

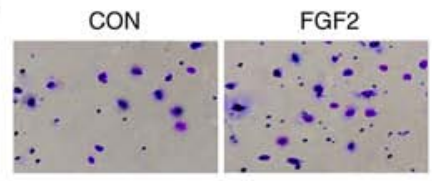

AZD4547 LY294002 $48 \mathrm{~h}$
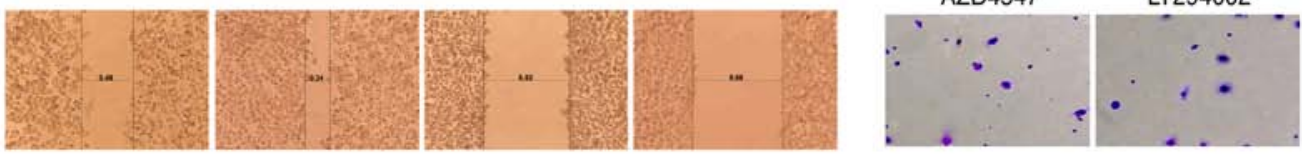

G
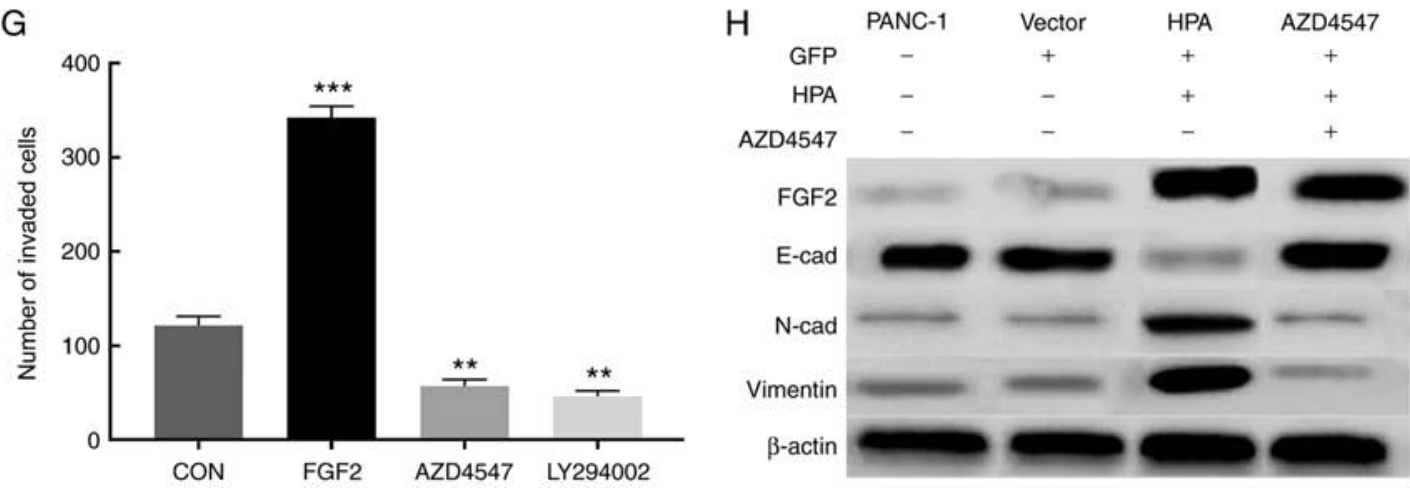

Figure 4. HPA promotes cell migration and invasion by activating the PI3K/Akt signaling pathway and EMT processes via FGF2 upregulation. (A) Expression of Palladin protein in various pancreatic cancer cell lines was analyzed by western blot assay. $\beta$-actin was used as an internal control. (B) Analysis of the relative expression of Palladin mRNA in pancreatic cancer cell lines by reverse transcription-quantitative PCR. (C) Western blot analysis of the effect of FGF2 on Palladin and Akt. (D) Protein expression of Palladin and Akt. $\beta$-actin was used as an internal control. (E) Effect of FGF2, AZD4547 and LY294002 on the migratory ability of PANC-1 cells assessed by wound healing assay. Magnification, $\mathrm{x} 200$. (F) Transwell assays were performed to determine the invasive ability of PANC-1 cells treated with FGF2, AZD4547 and LY294002. (G) Number of invaded PANC-1 cells treated with FGF2, AZD4547 and LY294002. CON, PANC-1 cell line transfected with empty plasmid. (H) Western blot analysis of the effect of HPA and FGF2 on EMT. Vector, PANC-1 cell line transfected with empty plasmid. $\beta$-actin was used as an internal control. ${ }^{*} \mathrm{P}<0.05,{ }^{* *} \mathrm{P}<0.01,{ }^{* * *} \mathrm{P}<0.001$. HPA, heparanase; FGF2, fibroblast growth factor 2; EMT, epithelial-mesenchymal transition; CON, PANC-1 cell line transfected with empty plasmid; Vector, PANC-1 cell line transfected with empty plasmid containing the GFP sequence.

the expression of FGF2 significantly decreased, a result which was consistent with the study hypothesis.

It has been reported that intracellular FGF2 signaling is activated by mitogen-activated protein kinases, PI3K/Akt and phosphatase $\mathrm{C} \gamma$ signaling pathways. In these pathways, PI3K/Akt signaling influences tumor cell invasion and migration, and is closely associated with FGF2-induced EMT $(33,34)$. Treatment of PANC-1 cells with FGF2 was found to significantly enhance cell migration and invasion.
By contrast, the invasion and migration of PANC-1 cells were markedly suppressed after treatment with the FGF2R inhibitor AZD4547 and the PI3K/Akt signaling pathway-specific inhibitor LY294002, respectively. The present results indicated that HPA is able to induce cell invasion and migration by upregulating FGF2, which in turn activates the PI3K/Akt signaling pathway in pancreatic cancer cells.

The cell scaffold protein Palladin serves an important role in wound healing, development of chronic inflammation 
and progression of malignant tumors $(35,36)$. A previous study showed that Palladin protein is highly expressed in breast cancer tissues, and its expression level is linked to the degree of invasion and metastasis of breast cancer (37). PI3K/Akt signaling induces the expression of Palladin, thereby enhancing cell motility, and promoting the migration and invasion of cancer cells (15). In the present study, PANC-1 cells were divided into groups, subjected to different interventions and compared. FGF2 was able to promote the expression of Palladin by activating the PI3K/Akt signaling pathway, a result which is consistent with the study hypothesis.

Previous reports have demonstrated that, during EMT activation, the expression of E-cadherin and certain cytokeratins (vimentin, fbronectin, twist and slug) is diminished, whereas the expression of mesenchymal markers is activated $(38,39)$. The most prominent mesenchymal markers are $\mathrm{N}$-cadherin, vimentin, fibronectin and integrins $(40,41)$. Western blot analysis was conducted to detect the expression of the epithelial phenotype marker E-cadherin, and the mesenchymal phenotype markers $\mathrm{N}$-cadherin and vimentin. According to the current results, using FGF2R inhibitor AZD4547 to prevent the binding of FGF2 to FGF2R, was capable of reversing the downregulation of E-cadherin and the upregulation of $\mathrm{N}$-cadherin and vimentin induced by HPA overexpression, thus inhibiting the activation of EMT. According to Thompson et al (42), EMT serves a major role in tumor development. In conclusion, it was hypothesized that HPA activates EMT by upregulating the expression of FGF2, thereby promoting the migration and invasion of pancreatic cancer cells.

However, the present study had certain limitations, including the small sample of matched paracancerous tissues, lack of correlation analysis between target gene/protein and prognosis and an absence of in vivo experiments. Future studies should further verify the relevant mechanisms, use animal models to confirm the relevant pathways and conduct prognostic correlation analysis.

In conclusion, the present study demonstrated that HPA and FGF2 are highly expressed in pancreatic cancer tissues and cells, and are closely associated with the clinicopathological characteristics of pancreatic cancer. Moreover, the HPA/SDC1 axis was observed to mediate the upregulation of FGF2, which in turn activated the EMT process, and promoted the invasion and migration of pancreatic cancer cells. The results of the present study indicate that the HPA/SDC1/FGF2 axis may be a valuable therapeutic target for pancreatic cancer.

\section{Acknowledgements}

The authors would like to thank Dr. Yumeng Jing (The First Affiliated Hospital of Shanxi Medical University, Taiyuan China) for critically reviewing the pathology results. The authors would also like to thank Mr. Yuan Jia of the general surgery laboratory teacher of Shanxi University Hospital (Taiyuan China), for his guidance and technical support.

\section{Funding}

The present study was supported by the Shanxi Province ' 136 ' Xingyi Medical Engineering Leading Specialist and Shanxi Basic Research Project (grant no. 2015011131).

\section{Availability of data and materials}

The datasets used and/or analyzed during the current study are available from the corresponding author on reasonable request.

\section{Authors' contributions}

HLZ and XC designed the study. XC, CC, JL and HCZ performed the experiments. XC wrote the paper. HLZ, JH and $\mathrm{XF}$ reviewed and edited the manuscript. JH and XF conducted statistical analysis of the data. All authors read and approved the final version of the manuscript.

\section{Ethics approval and consent to participate}

The present study was reviewed and ethically approved by the Shanxi Dayi Hospital Ethics Committee. Informed written consent was obtained from each patient and his/her family.

\section{Patient consent for publication}

Not applicable.

\section{Competing interests}

The authors declare that they have no competing interests.

\section{References}

1. Bray F, Ferlay J, Soerjomataram I, Siegel RL, Torre LA and Jemal A: Global cancer statistics 2018: GLOBOCAN estimates of incidence and mortality worldwide for 36 cancers in 185 countries. CA Cancer J Clin 68: 394-424, 2018.

2. McGuigan A, Kelly P, Turkington RC, Jones C, Coleman HG and McCain RS: Pancreatic cancer: A review of clinical diagnosis, epidemiology, treatment and outcomes. World J Gastroenterol 24: 4846-4861, 2018.

3. Puleo F, Maréchal R, Demetter P, Bali MA, Calomme A, Closset J, Bachet JB, Deviere J and Van Laethem JL: New challenges in perioperative management of pancreatic cancer. World J Gastroenterol 21: 2281-2293, 2015.

4. Szatmári T, Mundt F, Kumar-Singh A, Möbus L, Ötvös R, Hjerpe A and Dobra K: Molecular targets and signaling pathways regulated by nuclear translocation of syndecan-1. BMC Cell Biol 18: 34, 2017.

5. Ibrahim SA, Gadalla R, El-Ghonaimy EA, Samir O, Mohamed HT, Hassan H, Greve B, El-Shinawi M, Mohamed MM and Götte M: Syndecan-1 is a novel molecular marker for triple negative inflammatory breast cancer and modulates the cancer stem cell phenotype via the IL-6/STAT3, Notch and EGFR signaling pathways. Mol Cancer 16: 57, 2017.

6. Jung O, Trapp-Stamborski V, Purushothaman A, Jin H, Wang H, Sanderson RD and Rapraeger AC: Heparanase-induced shedding of syndecan-1/CD138 in myeloma and endothelial cells activates VEGFR2 and an invasive phenotype: Prevention by novel synstatins. Oncogenesis 5: e202, 2016.

7. Meirovitz A, Hermano E, Lerner I, Zcharia E, Pisano C, Peretz T and Elkin M: Role of heparanase in radiation-enhanced invasiveness of pancreatic carcinoma. Cancer Res 71: 2772-2780, 2011.

8. Quiros RM, Rao G, Plate J, Harris JE, Brunn GJ, Platt JL, Gattuso P, Prinz RA and Xu X: Elevated serum heparanase-1 levels in patients with pancreatic carcinoma are associated with poor survival. Cancer 106: 532-540, 2006.

9. Hegab AE, Ozaki M, Kameyama N, Gao J, Kagawa S, Yasuda H, Soejima K, Yin Y, Guzy RD, Nakamura Y, et al: Effect of FGF/FGFR pathway blocking on lung adenocarcinoma and its cancer-associated fibroblasts. J Pathol 249: 193-205, 2019.

10. La Venuta G, Zeitler M, Steringer JP, Müller HM and Nickel W: The startling properties of fibroblast growth factor 2: How to exit mammalian cells without a signal peptide at hand. J Biol Chem 290: 27015-27020, 2015. 
11. Masola V,Zaza G, Secchi MF, Gambaro G,Lupo A and Onisto M: Heparanase is a key player in renal fibrosis by regulating TGF- $\beta$ expression and activity. Biochim Biophys Acta 1843: 2122-2128, 2014.

12. Livak KJ and Schmittgen TD: Analysis of relative gene expression data using real-time quantitative PCR and the 2(-Delta Delta $\mathrm{C}(\mathrm{T})$ ) method. Methods 25: 402-408, 2001.

13. Yang Y, MacLeod V, Miao HQ, Theus A, Zhan F, Shaughnessy JD Jr, Sawyer J, Li JP, Zcharia E, Vlodavsky I and Sanderson RD: Heparanase enhances syndecan-1 shedding: A novel mechanism for stimulation of tumor growth and metastasis. J Biol Chem 282: 13326-13333, 2007.

14. Goicoechea SM, García-Mata R, Staub J, Valdivia A, Sharek L, McCulloch CG, Hwang RF, Urrutia R, Yeh JJ, Kim HJ and Otey CA: Palladin promotes invasion of pancreatic cancer cells by enhancing invadopodia formation in cancer-associated fibroblasts. Oncogene 33: 1265-1273, 2014.

15. Chin YR and Toker A: Akt2 regulates expression of the actin-bundling protein Palladin. FEBS Lett 584: 4769-4774, 2010.

16. Bame KJ: Heparanases: Endoglycosidases that degrade heparan sulfate proteoglycans. Glycobiology 11: 91R-98R, 2001.

17. Nasser NJ: Heparanase involvement in physiology and disease. Cell Mol Life Sci 65: 1706-1715, 2008.

18. Purushothaman A, Uyama T, Kobayashi F, Yamada S, Sugahara K, Rapraeger AC and Sanderson RD: Heparanase-enhanced shedding of syndecan-1 by myeloma cells promotes endothelial invasion and angiogenesis. Blood 115: 2449-2457, 2010.

19. Okawa T, Naomoto Y, Nobuhisa T, Takaoka M, Motoki T, Shirakawa Y, Yamatsuji T, Inoue H, Ouchida M, Gunduz M, et al Heparanase is involved in angiogenesis in esophageal cancer through induction of cyclooxygenase-2. Clin Cancer Res 11: 7995-8005, 2005.

20. Masola V, Zaza G, Onisto M, Lupo A and Gambaro G: Impact of heparanase on renal fibrosis. J Transl Med 13: 181, 2015.

21. Gingis-Velitski S, Zetser A, Kaplan V, Ben-Zaken O, Cohen E, Levy-Adam F, Bashenko Y, Flugelman MY, Vlodavsky I and Ilan N: Heparanase uptake is mediated by cell membrane heparan sulfate proteoglycans. J Biol Chem 279: 44084-44092, 2004.

22. Meirovitz A, Goldberg R, Binder A, Rubinstein AM, Hermano E and Elkin $M$ : Heparanase in inflammation and inflammation-associated cancer. FEBS J 280: 2307-2319, 2013.

23. Sanderson RD, Elkin M, Rapraeger AC, Ilan N and Vlodavsky I: Heparanase regulation of cancer, autophagy and inflammation: New mechanisms and targets for therapy. FEBS J 284: 42-55, 2017

24. Rugo HS, Delord JP, Im SA, Ott PA, Piha-Paul SA, Bedard PL Sachdev J, Tourneau CL, van Brummelen EMJ, Varga A, et al: Safety and antitumor activity of pembrolizumab in patients with estrogen receptor-positive/human epidermal growth factor receptor 2-negative advanced breast cancer. Clin Cancer Res 24: 2804-2811, 2018

25. Qu Y, Zhang H, Sun W, Han Y, Li S, Qu Y, Ying G and Ba Y: MicroRNA-155 promotes gastric cancer growth and invasion by negatively regulating transforming growth factor- $\beta$ receptor 2 . Cancer Sci 109: 618-628, 2018

26. Nandy D and Mukhopadhyay D: Growth factor mediated signaling in pancreatic pathogenesis. Cancers (Basel) 3: 841-871, 2011.
27. Khurana A, Liu P, Mellone P, Lorenzon L, Vincenzi B, Datta K, Yang B, Linhardt RJ, Lingle W, Chien J, et al: HSulf-1 modulates FGF2- and hypoxia-mediated migration and invasion of breast cancer cells. Cancer Res 71: 2152-2161, 2011.

28. Li T, Meng XL and Yang WQ: Long noncoding RNA PVT1 acts as a 'Sponge' to inhibit microRNA-152 in gastric cancer cells. Dig Dis Sci 62: 3021-3028, 2017.

29. Coleman SJ, Chioni AM, Ghallab M, Anderson RK, Lemoine NR Kocher HM and Grose RP: Nuclear translocation of FGFR1 and FGF2 in pancreatic stellate cells facilitates pancreatic cancer cell invasion. EMBO Mol Med 6: 467-481, 2014.

30. Kleeff J, Friess H, Buechler M, Kothari N, Datta S, Fan H and Korc M: Adenovirus mediated transfer of a truncated fibroblast growth factor (FGF) typ I receptor blocks FGF2 signaling in pancreatic cancer cells. Gastroenterology 124: A104, 2003.

31. Li RW, Freeman C, Yu D, Hindmarsh EJ, Tymms KE, Parish CR and Smith PN: Dramatic regulation of heparanase activity and angiogenesis gene expression in synovium from patients with rheumatoid arthritis. Arthritis Rheum 58: 1590-1600, 2008.

32. Kato M, Wang H, Kainulainen V, Fitzgerald ML, Ledbetter S, Ornitz DM and Bernfield M: Physiological degradation converts the soluble syndecan-1 ectodomain from an inhibitor to a potent activator of FGF-2. Nat Med 4: 691-697, 1998.

33. Dailey L, Ambrosetti D, Mansukhani A and Basilico C: Mechanisms underlying differential responses to FGF signaling. Cytokine Growth Factor Rev 16: 233-247, 2005.

34. Katoh $\mathrm{M}$ and Katoh M: Cross-talk of WNT and FGF signaling pathways at GSK3beta to regulate beta-catenin and SNAIL signaling cascades. Cancer Biol Ther 5: 1059-1064, 2006.

35. Boukhelifa M, Hwang SJ, Valtschanoff JG, Meeker RB, Rustioni A and Otey CA: A critical role for Palladin in astrocyte morphology and response to injury. Mol Cell Neurosci 23: 661-668, 2003.

36. Najm P and El-Sibai M: Palladin regulation of the actin structures needed for cancer invasion. Cell Adh Migr 8: 29-35, 2014

37. Goicoechea SM, Bednarski B, García-Mata R, Prentice-Dunn H, $\mathrm{Kim} \mathrm{HJ}$ and Otey CA: Palladin contributes to invasive motility in human breast cancer cells. Oncogene 28: 587-598, 2009.

38. Yilmaz M and Christofori G: EMT, the cytoskeleton, and cancer cell invasion. Cancer Metastasis Rev 28: 15-33, 2009.

39. Brabletz T, Kalluri R, Nieto MA and Weinberg RA: EMT in cancer. Nat Rev Cancer 18: 128-134, 2018.

40. Shibue T and Weinberg RA: EMT, CSCs, and drug resistance: The mechanistic link and clinical implications. Nat Rev Clin Oncol 14: 611-629, 2017.

41. Lamouille S, Xu J and Derynck R: Molecular mechanisms of epithelial-mesenchymal transition. Nat Rev Mol Cell Biol 15: 178-196, 2014.

42. Thompson EW, Newgreen DF and Tarin D: Carcinoma invasion and metastasis: A role for epithelial-mesenchymal transition? Cancer Res 65: 5991-5995, 2005.

(i) $\Theta$ This work is licensed under a Creative Commons Attribution-NonCommercial-NoDerivatives 4.0 International (CC BY-NC-ND 4.0) License. 